\title{
Utilização do algoritmo de árvore de decisão para mapeamento do uso e cobertura da terra da área de contribuição de uma barragem localizada na Bacia Hidrográfica do São Francisco
}

\author{
Mariana Faria Veloso ${ }^{1 *}$; Rodolpho César Tinini dos Reis²; Raul de Magalhães Filho ${ }^{3}$; Flávia Mazzer Rodrigues ${ }^{4}$
}

DOI: https://doi.org/10.35699/2447-6218.2021.29039

\begin{abstract}
Resumo
O objetivo deste estudo é mapear o uso e cobertura da terra da área de contribuição da barragem Bico da Pedra através do algoritmo árvore de decisão. Para isso, foram adquiridas o modelo de elevação SRTM e duas imagens (verão e inverno) do satélite Landsat 8 sensor OLI/TIRS, ano 2017. Em seguida, realizou-se o cálculo dos índices NDVI e SAVI, e então inseriu um conjunto de onze variáveis na árvore de decisão resultando em um mapa de uso e cobertura da terra com nove classes: cerrado, floresta decidual montana, campos, água, pasto/solo exposto, culturas, afloramento rochoso, barragem de rejeitos e mancha urbana. A classe mais expressiva verificada na área de estudo foi a vegetação correspondendo em quase $80 \%$. Além disso, foi verificado presença de culturas próximas à nascente do rio Gorutuba e ao reservatório da barragem. A classe de pasto/solo exposto apresentou em quase metade de suas amostras como equivocadas. A acurácia da classificação foi satisfatória e o Índice Kappa, excelente. Portanto, os resultados apresentados permitiram adquirir informações de uso e cobertura do solo capazes de auxiliar na gestão e planejamento dos recursos hídricos e ambientais da área de contribuição da barragem Bico da Pedra.
\end{abstract}

Palavras-chave: Classificação de imagens. Geotecnologias. Landsat 8.

\section{Application of the decision tree algorithm to map the land use/land cover of the contribution area of a dam located in the São Francisco River Basin}

\begin{abstract}
The aim of this study is to map the land use and cover of the contribution area of the Bico da Pedra dam through the decision tree algorithm. For this, the SRTM elevation model and two images (summer and winter) from the Landsat 8 satellite OLI / TIRS sensor, year 2017 were acquired. Then, the NDVI and SAVI indices were calculated, and then a set of eleven variables in the decision tree and in a land use and land cover map with nine classes: cerrado, montane deciduous forest, fields, water, pasture / exposed soil, crops, rocky outcrop, tailings dam and urban. The most expressive class verified in the study area was the vegetation corresponding to almost $80 \%$. In addition, the presence of crops close to the source of the Gorutuba river was verified. The class of pasture / exposed soil presented in almost half of its samples as mistaken. The accuracy of the classification was satisfactory and the Kappa Index excellent. Therefore, the results presented allowed to acquire information on land use and cover capable of assisting in the management and planning of water and environmental resources in the area of contribution of the Bico da Pedra dam.
\end{abstract}

Keywords: Image classification. Geotechnologies. Landsat 8.

\footnotetext{
${ }^{1}$ Universidade Federal de Viçosa, Departamento de Engenharia Agrícola, Viçosa, MG, Brasil.

https://orcid.org/0000-0002-8368-7877

${ }^{2}$ Universidade Federal de Minas Gerais, Instituto de Ciências Agrárias, Montes Claros, MG, Brasil. https://orcid.org/0000-0003-2052-5368

${ }^{3}$ Universidade Estadual de Montes Claros, Montes Claros, MG, Brasil. https://orcid.org/0000-0003-0131-5453

${ }^{4}$ Universidade Federal de Minas Gerais, Instituto de Ciências Agrárias, Montes Claros, MG, Brasil.

https://orcid.org/0000-0001-5520-1844

*Autor para correspondência: mah_veloso7@hotmail.com
}

Recebido para publicação em 28 de janeiro de 2021. Aceito para publicação em 16 de março de 2021. e-ISSN: 2447-6218 / ISSN: 2447-6218. Atribuição CC BY. 


\section{Introdução}

As discussões resultantes da interação entre as atividades humanas e o meio ambiente tornaram-se intensas nos últimos anos, gerando preocupações junto às questões ambientais, econômicas e sociais, presentes e futuras. A exploração inadequada dos recursos naturais é a maior preocupação, principalmente quando se refere às transformações da ação antrópica que em muitas situações são irreversíveis.

Diante desse contexto, a análise de uso e ocupação territorial se torna fundamental para a gestão e planejamento dos recursos naturais e as bacias hidrográficas tornam-se as unidades de estudo, conforme a Lei 9.433, de 8 de janeiro de 1997. Considerada uma importante bacia hidrográfica brasileira, a Bacia Hidrográfica do Rio São Francisco (BHRSF) já apresenta condições adversas, com vazões e precipitações abaixo da média prejudicando os níveis de armazenamento dos reservatórios ali presentes (CBHSF, 2016).

Inserida em parte na BHSF, a região do norte de Minas Gerais se identifica a intensa exploração dos recursos hídricos, mesmo com os longos períodos de estiagens e baixa disponibilidade hídrica superficial (CPRM, 2019). Um dos rios de maior importância dessa região, é o rio Gorutuba, onde se encontra a barragem Bico da Pedra responsável pelo abastecimento de água dos municípios de Janaúba, Porteirinha e Nova Porteirinha, além do fornecimento de água para áreas irrigáveis (DIG, 2013). Contudo, a presença de barragens em vales fluviais rompe com a sequência natural dos cursos d'água, gerando impactos ambientais nas áreas de contribuição que se estendem a montante do rio (Coelho, 2008).

Com a finalidade de obter maior compreensão dos processos que impactam o meio ambiente e as alternativas para mitigá-los, foram desenvolvidas geotecnologias capazes de realizar o monitoramento e análises ambientais a partir de dados espaciais, como o Sistema de Informação Geográfica (SIG) e o sensoriamento remoto (Martins et al., 2014; Rosa, 2005). Essa relação possibilita a criação de mapas de uso e cobertura da terra, utilizados na identificação e interpretação de alvos como áreas degradadas, cultivos, vegetações, cursos d água e outras feições, bem como analisar temporariamente as paisagens provocadas pela ação humana (Santos; Petronzio, 2011)

A classificação desses alvos pode ser feita por meio de diferentes métodos, entre eles, a árvore de decisão, algoritmo de aprendizagem com estrutura composta pelos nós, ramos e folhas que permite analisar um conjunto de atributos com base em decisões booleanas, que são representadas na saída por um valor ou rótulo (Berhane et al., 2018). A classificação pela árvore de decisão possui vantagens sobre outros classificadores, pois apresentam uma semântica praticamente intuitiva, de fácil compreensão, simples e flexível, além de apresentar acurácias superiores à classificadores como Máquina de
Vetor de Suporte e Máxima Verossimilhança (Yang et al., 2017)

Diante desse contexto, o objetivo do presente estudo é, por meio do algoritmo de árvore de decisão, mapear o uso e cobertura da terra da área de contribuição da barragem Bico da Pedra, norte de Minas Gerais, com vistas a identificação das suas fragilidades ambientais.

\section{Material e métodos}

\section{Área de estudo}

A área de estudo foi definida como a área de contribuição da barragem Bico da Pedra, localizada entre os municípios de Francisco Sá, Janaúba, Porteirinha, Nova Porteirinha e Riacho dos Machados, pertencente à Bacia Hidrográfica do Rio Verde Grande, afluente do rio São Francisco, situados na região do norte do estado de Minas Gerais, Brasil (Figura 1).

Os municípios que compreendem a área de estudo apresentam clima tropical, sendo o verão mais chuvoso que o inverno, configurando como classificação Aw (Köppen, 1936). A temperatura e pluviosidade média desses munícipios são de $23,7^{\circ} \mathrm{C}$ e $830 \mathrm{~mm}$, respectivamente (INMET, 2018).

Os tipos de solos presentes na área de contribuição da barragem Bico da Pedra, segundo a FEAM (2010), correspondem em sete classes: afloramento rochoso, argissolo vermelho-amarelo, cambissolo háplico, latossolo vermelho, latossolo vermelho-amarelo, neossolo litólico distrófico e nitossolo vermelho.

\section{Aquisição e processamento dos dados}

Inicialmente, realizou-se a delimitação da área de contribuição da barragem Bico da Pedra a partir do modelo de elevação Shuttle Radar Topography Mission (SRTM) de resolução espacial 30 metros. Posteriormente, foram adquiridas duas imagens do satélite Landsat 8 sensor OLI (Operational Land Imager) e TIRS (Thermal Infrared Sensor), referentes ao ano de 2017, para os períodos de verão (08/01/2017) e inverno (04/08/2017). Além disso, foi realizada a correção atmosférica das imagens de satélite através do algoritmo DOS1 (Dark Object Substraction 1) (Chavez Junior, 1988).

Os índices de vegetação Normalized Difference Vegetation Index (NDVI) (Rouse et al., 1973) e Soil Adjusted Vegetation Index (SAVI) (Huete, 1988) foram calculados, conforme a equações 1 e 2 , respectivamente. Sendo que, o SAVI considera os efeitos do solo, de forma à ajustar o NDVI em superfícies não cobertas pela vegetação. 


$$
\begin{gathered}
\text { NDVI }=\frac{\mathrm{B}_{\text {Infravermelho }}-\mathrm{B}_{\text {Vermelho }}}{\mathrm{B}_{\text {Infravermelho }}+\mathrm{B}_{\text {Vermelho }}} \text { (Eq. 1) } \\
\text { SAVI }=\frac{\left(\mathrm{B}_{\text {Infravermelho }}-\mathrm{B}_{\text {Vermelho }}\right)}{\left(\mathrm{B}_{\text {Infravermelho }}+\mathrm{B}_{\text {Vermelho }}+\mathrm{L}\right)}(1+\mathrm{L}) \quad(\text { Eq. } 2)
\end{gathered}
$$

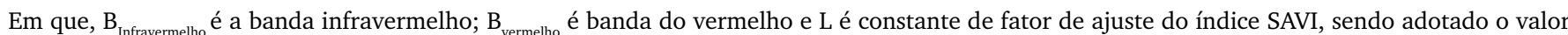
de 0,5 , pois a vegetação da área de estudo é de densidade intermediária (Huete, 1988).

Figura 1 - Localização da área de contribuição da barragem Bico da Pedra no contexto regional e do estado de Minas Gerais

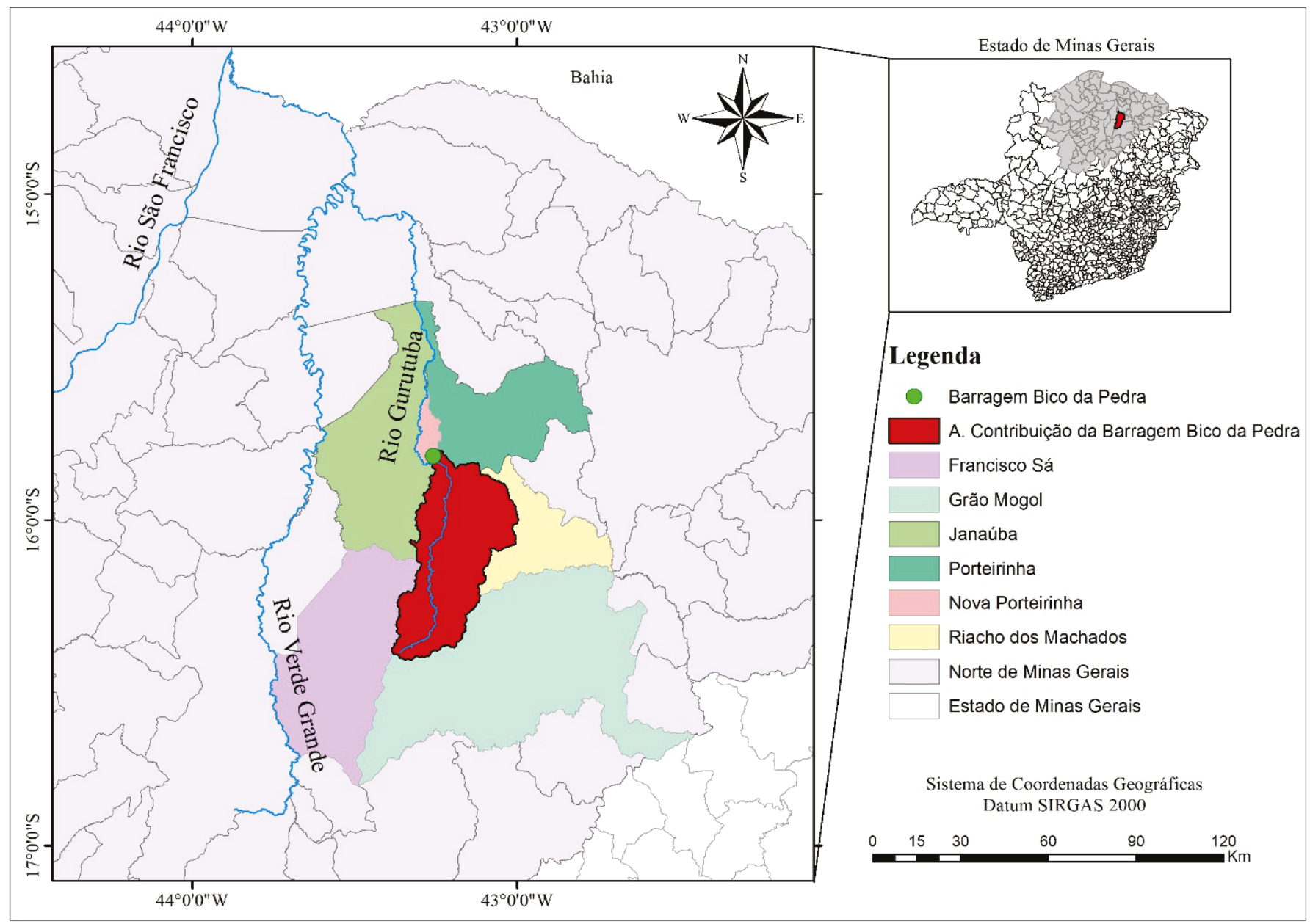

\section{Criação da árvore de decisão}

Para a criação da árvore decisão utilizou-se a ferramenta Decision Tree do software ENVI 5.3. A primeira variável incorporada na árvore, representando o primeiro nó raiz de decisão, foi o limite da área de estudo, ou seja, a área de contribuição da barragem Bico da Pedra. Essa variável permite que a partir dessa etapa, todas variáveis a serem introduzidas na árvore de decisão não extrapolem o limite da área de estudo.

Em seguida, foram criadas máscaras de limites de determinadas classes utilizando o termo "Mask" para referenciar à essas feições, sendo essas: Limite_Mask (Nó raiz), Urbano_Mask associado a mancha urbana do município de Riacho dos Machados, Rejeito_Mask que compreende a barragem de rejeitos presente na área de estudo e Cultura_Mask referenciando aos cultivos.
Posteriormente, foram estabelecidos os limiares das equações de decisões (booleanas) para as variáveis índices de vegetação (NDVI e SAVI), altimetria (através do modelo SRTM) e para as bandas do vermelho (B4), infravermelho próximo (B5) e o infravermelho médio (B6). Para isso, foram realizadas coletas de 50 pixels para cada uma dessas variáveis e extraindo a média desses valores, sendo que para as bandas espectrais foram coletadas as amostras para cada classe de uso e cobertura do solo identificado na área de estudo: tipos de vegetação, solo exposto, pasto e corpos hídricos.

A Tabele 1 apresenta as onze variáveis $\left(\mathrm{X}_{1}, \mathrm{X}_{2}, \mathrm{X}_{3}\right.$, ..., $\mathrm{X}_{11}$ ) e seus respectivos limiares das equações lógicas utilizados para a criação da árvore de decisão. 
Veloso, M. F. et al.

Figura 2 - Tipos de solo presentes na área de contribuição da barragem Bico da Pedra

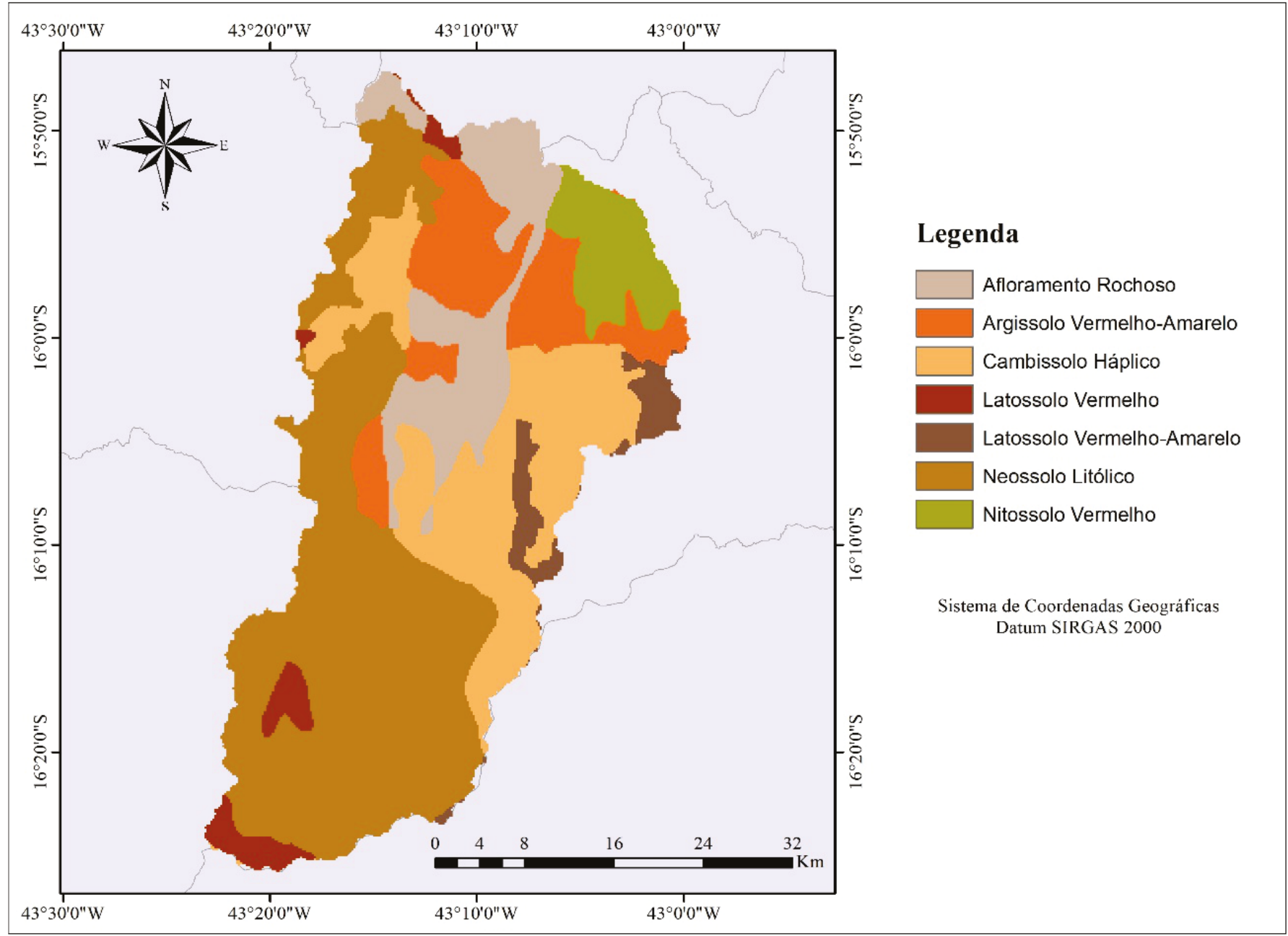

Tabela 1 - Variáveis e suas respectivas equações lógicas utilizadas para a construção da árvore de decisão

\begin{tabular}{lc}
\hline Variável & Equação \\
\hline Limite_Mask & $\mathrm{X}_{1}$ eq 0 \\
Culturas_Mask & $\mathrm{X}_{2}$ eq 0 \\
Rejeito_Mask & $\mathrm{X}_{3}$ eq 0 \\
Urbano_Mask & $\mathrm{X}_{4}$ eq 0 \\
NDVI & $\mathrm{X}_{5}$ ge 0.3 \\
SAVI & $\mathrm{X}_{6}$ ge 0.34 \\
SRTM & $\mathrm{X}_{7}$ ge 1100 \\
L8_V_B5 & $\mathrm{X}_{8}$ le 0.29 \\
L8_I_B5 & $\mathrm{X}_{9}$ le 0.21 \\
L8_V_B4 & $\mathrm{X}_{10}$ le 0.103 \\
L8_V_B6 & $\mathrm{X}_{11}$ ge 0.55 \\
\hline
\end{tabular}

L8 = Landsat 8; V = verão; $\mathrm{I}=$ inverno; $\mathrm{X}_{\mathrm{n}}$ = variável; eq = igual; ge = maior que; le = menor que.

Vale ressaltar que, para distinção dos tipos de vegetação, foi fundamental a coleta dos pixels nos dois períodos trabalhados, verão e inverno. Além disso, a escolha das bandas B4, B5 e B6 são devido a melhor capacidade de resposta espectral da vegetação nesses comprimentos de ondas (Jensen, 2015). 
A finalização do procedimento ocorreu quando se atingiu uma distinção adequada das classes estabelecidas. da terra da área de contribuição da barragem Bico da Pedra é apresentada na Figura 3.

O fluxograma construído através do algoritmo árvore de decisão, para classificação de uso e cobertura

Figura 3 - Fluxograma da árvore de decisão utilizada para o mapeamento da área de estudo, a partir da ferramenta Decision Tree do software ENVI 5.3

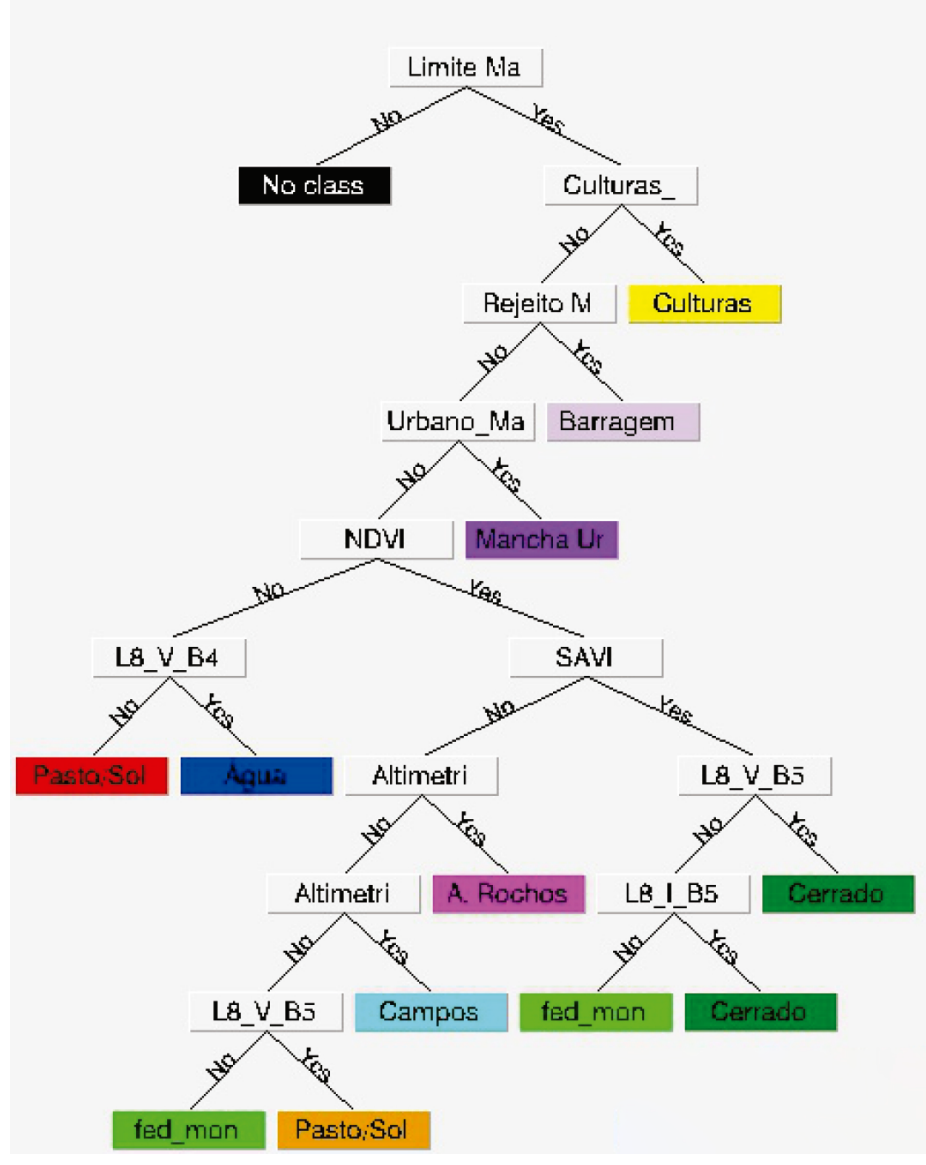

Visando estabelecer uma melhor precisão das classes estabelecidas para área de estudo, teve-se a combinação de onze variáveis para distinguir nove classes: cerrado, floresta estacional decidual montana (FED_mon), campos, corpos hídricos, pasto/solo exposto, cultura, afloramento rochoso, mancha urbana e barragem de rejeitos.

O nó raiz, a variável limite_mask, definiu-se como a área de interesse, sendo que os valores iguais a zero indicaram a classe "no class", ou seja, não compreende o interior do limite da área de estudo. Em seguida as máscaras mancha_urbana, barragem_rejeitos e culturas foram introduzidos na árvore.

Na etapa seguinte, utilizou a variável NDVI e os valores médios dos pixels maiores ou iguais a 0.3 foram classificados com áreas vegetadas e os valores menores que 0.3 como não vegetados ou referente a outra vegetação. Para o ramo negativo foi utilizado a banda 4 do período de verão para definir melhor a classe água. $\mathrm{Na}$ divisão do ramo positivo do NDVI, a variável SAVI foi empregada com o valor limiar maior ou igual a 0.34 classificado como cerrado e o valor menor que 0.34 como demais classes.

No ramo negativo, a variável altimetria foi utilizada a partir do modelo de elevação SRTM e o valor limiar superior a 1100 metros de altitude, destina-se área de afloramento rochoso e superior a 1000 metros são os campos. A variável L8_V_B5 definiu as classes de pasto/ solo exposto e Floresta Estacional Decidual Montana (FED mont). Já no ramo positivo, empregou a variável L8_V_B5 com um limiar inferior de 0.29 para cerrado e também recorreu a variável L8 I B5 do período de inverno com valor inferior a 0.21 para caracterizar melhor o cerrado e o ramo negativo foram caracterizados como FED_mont.

Pode-se observar a aplicação repetitiva de algumas variáveis, demostrando a heterogeneidade da área de estudo, por exemplo a variável altimetria utilizada para separar dois fragmentos, um de solo e outro de vegetação. 
Veloso, M. F. et al.

\section{Resultados e discussão}

O resultado da classificação supervisionada de uso e cobertura da terra, gerada através do algoritmo de árvore de decisão, referente ao ano de 2017 da área de contribuição da Barragem Bico da Pedra está apresentado na Figura 4.

Figura 4 - Mapa de uso e cobertura da terra da área de contribuição da barragem Bico da Pedra

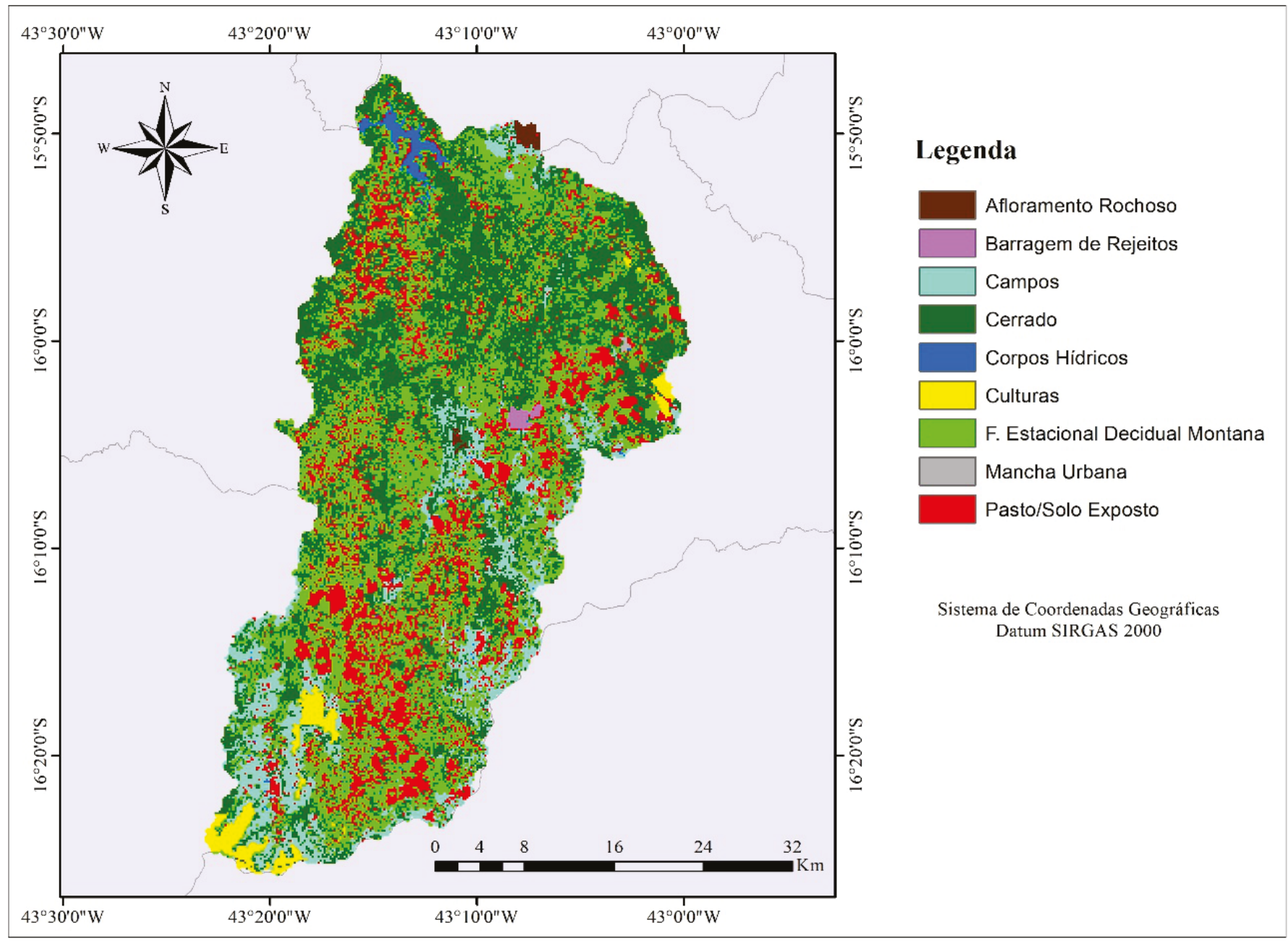

A maior parte da área de contribuição da barragem Bico da Pedra é composta pela vegetação, seja cerrado, floresta estacional decidual montana ou campos. Essa caracterização pode ser verificada no Mapa de Cobertura Vegetal (ZEE, 2009), em que as áreas de vegetação dentro da área de contribuição da barragem apresenta em grande parte a vegetação de cerrado, floresta estacional decidual montana e campos.

As áreas de pasto/solo exposto foram colocadas em uma única classe devido a dificuldade em distingui-las. Ao observar a predominância do tipo de solo na área de estudo, as classes Cambissolo háplico e Neossolo litólico são as mais significativas. Além disso, as áreas de pastagens e solos expostos, equivalentes a $270.46 \mathrm{~km}^{2}(16.65$ $\%$ da área total), se encontram em sua maior parte nessa região (Tabela 2). Segundo a Embrapa (2006), os tipos Cambissolos e Neossolos são solos de baixa fertilidade, o que favorece a atividade do pasto, que consequentemente pode desenvolver-se à solos expostos, isso dependendo da não prática de ações conservacionistas de solo e água.
As áreas de culturas apresentam predominância de cultivos de eucalipto, aspecto verificado pela homogeneidade e imagens do programa Google Earth Pro. O cultivo de eucalipto depende de diversos fatores como clima, tipo de solo, sistema de cultivo e entre outros, o que favorece a escolha do eucalipto ideal e o aumento da produtividade (Embrapa, 2014).

Contudo, ao analisar onde se encontram as áreas mais extensas de eucalipto (porção sul da área de contribuição da barragem Bico da Pedra), é verificada a proximidade dos cultivos à nascente do rio Gorutuba, o que pode comprometer as vazões ao longo do rio principal e seus afluentes. Em relação aos impactos ambientais dos cultivos, vale destacar que os conhecimentos de dados climatológicos (temperatura, precipitação e umidade relativa do ar) e tipo de solo são indispensáveis para a conservação da água e solo, sobretudo, os estudos de balanço hídrico entre a quantidade consumida de água pela planta e a precipitação pluviométrica da região. 
Utilização do algoritmo de árvore de decisão para mapeamento do uso e cobertura da terra da área de contribuição de uma barragem localizada na Bacia Hidrográfica do São Francisco

Tabela 2 - Classes de uso e cobertura da terra identificadas na área de estudo e seus respectivos valores de área.

\begin{tabular}{lcc}
\hline Classes de uso e cobertura da terra & Área $\left(\mathbf{k m}^{2}\right)$ & Área (\%) \\
\hline Corpos Hídricos & 18,68 & 1,15 \\
Barragem de Rejeitos & 4,06 & 0,25 \\
Culturas & 32,49 & 2,00 \\
Mancha Urbana & 0,82 & 0,05 \\
Pasto /Solo Exposto & 270,46 & 16,65 \\
Campos & 170,56 & 10,5 \\
FED_mon & 602,68 & 37,1 \\
Cerrado & 524,7 & 32,3 \\
\hline Total & 1624,45 & 100 \\
\hline
\end{tabular}

Leite, Dias e Rocha (2015) constataram por meio do sensoriamento remoto e visitas à campo, a diminuição do nível de água da barragem Bico da Pedra, sendo justificado pelo crescente consumo de água e às baixas precipitações nos últimos anos no norte de Minas Gerais. Os autores ainda verificaram impactos ambientais no entorno do reservatório como o aumento das rodovias e o desmatamento para a criação das mesmas ou para plantio de culturas, promovendo uma maior erosão do solo, e consequentemente um maior assoreamento do rio.

As mudanças na dinâmica de uso e cobertura do solo em áreas com presença de barragens também foram verificadas por Oliveira, Sá e Leite (2016), onde constatou-se que a vegetação do cerrado está reduzindo, enquanto aumentam as áreas de plantio de eucaliptos e atividades pastoris na área de drenagem da barragem de Juramento, norte de Minas Gerais, apesar da predominância da vegetação natural.

As classes de vegetação da área de contribuição da barragem Bico da Pedra correspondem quase $80 \%$ da área total, sendo os tipos dominantes o cerrado $(524,7$ $\mathrm{km}^{2}$ ) e a floresta estacional decidual montana $(602,68$ $\mathrm{km}^{2}$ ) (Tabela 3). A árvore de decisão possibilitou diferenciar bem os tipos de vegetação presentes na área de estudo, demostrando uma flexibilidade do classificador como pode ser observado por Almeida (2016), em que realiza o mapeamento de áreas de veredas na Área de Proteção Ambiental (APA) situadas no rio Pandeiros, norte de Minas Gerais. $\mathrm{O}$ autor utilizou, além da árvore de decisão, o algoritmo de Máxima Verossimilhança (MAXVER), sendo que este não obteve uma distinção satisfatória das veredas e já a árvore de decisão, distinguiu as veredas e outros tipos de vegetação, enfatizando a contribuição significativa das máscaras vetoriais e os índices de vegetação na criação da árvore de decisão.

\section{Avaliação da acurácia da classificação do uso e co- bertura da terra}

Visando avaliar a qualidade do mapeamento do uso e cobertura da terra da área de contribuição da barragem Bico da Pedra, foram obtidos a partir da matriz de erros, calculando a Acurácia Geral e o Indice Kappa. O mapa de uso e cobertura da terra da área de contribuição da barragem Bico da Pedra foi qualificado como excelente, com Acurácia Geral de 88,33 \% e Índice Kappa, valor de 0,8404, conforme Landis e Koch (1977).

Na Tabela 3 são apresentadas as estatísticas de acurácia do produtor, usuário e erros de comissão e omissão da classificação.

De acordo com a Tabela 5, a classificação obtida pelo algoritmo árvore de decisão apresenta um desempenho pior para a classe pasto/solo exposto, com a exatidão do usuário de $47.01 \%$ indicando que quase metade das amostras classificadas como pasto/solo exposto foram equivocadas. Esse fato pode estar relacionado a própria dificuldade de distinguir as classes de pasto e o solo exposto na área de estudo, na qual o tipo de solo verificado nessas classes são de baixa profundidade e teor de matéria orgânica, apresentando intensidades de reflectância altas como em solos expostos como pode ser verificado por Sousa Junior, Demattê e Genú (2008) e Almeida (2016).

Além disso, a exatidão do produtor para as classes de vegetação cerrado, afloramento rochoso e culturas apresentaram os melhores resultados com valores acima de $90 \%$. Isso, pode ser explicado pela facilidade visual ao coletar as amostras da classe cerrado e afloramento e pela criação das máscaras para a classe culturas, reduzindo os erros na classificação. 
Veloso, M. F. et al.

Tabela 3 - Acurácia do resultado da classificação de uso e cobertura da terra

\begin{tabular}{lcccc}
\hline & $\begin{array}{c}\text { Acurácia Produtor } \\
(\mathbf{\%})\end{array}$ & $\begin{array}{c}\text { Acurácia Usuário } \\
\mathbf{( \% )}\end{array}$ & $\begin{array}{c}\text { Erro de Comissão } \\
\mathbf{( \% )}\end{array}$ & $\begin{array}{c}\text { Erro de Omissão } \\
\text { (\%) }\end{array}$ \\
\hline Cerrado & 92,73 & 92,62 & 7,38 & 7,27 \\
FED_mon & 77,59 & 79,60 & 20,40 & 22,41 \\
Campos & 73,91 & 83,33 & 16,67 & 26,09 \\
Culturas & 93,93 & 98,82 & 1,18 & 6,07 \\
Afloramento Rochoso & 98,46 & 91,43 & 8,57 & 1,54 \\
Pasto/Solo Exposto & 46,74 & 47,01 & 52,99 & 53,26 \\
Corpos Hídricos & 81,13 & 93,48 & 6,52 & 18,87 \\
Mancha Urbana & 78,57 & 100,00 & 0,00 & 21,43 \\
Barragem Rejeitos & 100,00 & 100,00 & 0,00 & 0,00 \\
\hline
\end{tabular}

\section{Conclusão}

A metodologia empregada para a classificação do uso e cobertura da terra da barragem Bico da Pedra apresentou resultados consistentes através do algoritmo árvore de decisão, resultado comprovado pela acurácia de $88,33 \%$ e o coeficiente Kappa, considerado excelente.

Observou-se que cerca $80 \%$ da área de estudo é ocupada pela vegetação, em maior predominância a floresta estacional decidual montana e o cerrado, tendo sua distinção facilitada pelo uso de imagens referentes à períodos diferentes (verão e inverno), pelas variáveis índices de vegetação (NDVI e SAVI) e as bandas do vermelho e infra-vermelho-médio do satélite Landsat 8 sensor OLI e TIRS.

A classe de pasto/solo exposto apresentou uma área significativa mas foi a classe com os maiores erros, devido à dificuldade de diferenciação entre o pasto e o solo exposto, no entanto, não inviabilizou a metodologia empregada.

Os corpos hídricos classificados foram identificados em sua maioria dentro do reservatório, o que indica um baixo conteúdo de água ao longo do rio Gorutuba causado pela seca e assoreamento do curso d'água.

A presença de cultivos de eucalipto próximo a nascente do rio Gorutuba foi identificada, fazendo-se necessário estudos para analisar a ação dessa atividade florestal na quantidade e qualidade do curso d'água.

Dessa forma, verifica-se a importância das ferramentas do SIG e do sensoriamento remoto para a gestão e planejamento dos recursos hídricos e ambientais, como constatado nos resultados apresentados desse estudo, possibilitando a aquisição de informações sobre o uso e cobertura da terra da área de contribuição da barragem Bico da Pedra.

\section{Referências}

Almeida, J. W. L. 2016. Métodos de sensoriamento remoto no mapeamento de veredas na APA rio pandeiros. Belo Horizonte: Universidade Federal de Minas Gerais, 83f. Dissertação Mestrado. Disponível em: https:// repositorio.ufmg.br/handle/1843/IGCM-ADMP85.

Brasil. Lei n. 9.433, de 8 de janeiro de 1997. Política Nacional dos Recursos Hídricos. Disponível em: http://www.planalto.gov.br/ccivil_03/ leis/L9433.htm.

Berhane, T. M.; Lane, C. R.; Wu, Q.; Autrey, B. C.; Anenkhonov, O. A.; Chepinoga, V. V.; Liu, H. 2018. Decision-tree, rule-based, and random forest classification of high-resolution multispectral imagery for wetland mapping and inventory. Remote Sensing, 10:4. Doi: https:// doi.org/10.3390/rs10040580.

CBHSF - Comitê da bacia hidrográfica do rio São Francisco. Plano de recursos hídricos da bacia hidrográfica do rio São Francisco 2016/2025. 2016. Disponível em: https://cbhsaofrancisco.org.br/documentacao/ plano-de-recursos-hidricos-2016-2025/.
Chavez Junior, P. S. 1988. An improved dark-object subtraction technique for atmospheric scattering correction of multispectraldata. Remote Sensing of Environment, 24, 459-479. Doi: https://doi. org/10.1016/0034-4257(88)90019-3.

Coelho, A. L. N. 2008. Geomorfologia fluvial de rios impactados por barragens. Caminhos de Geografia, 9:26, 16-32. Doi: http://www.seer. ufu.br/index.php/caminhosdegeografia/article/view/15721.

CPRM - Serviço Geológico do Brasil. Projeto águas do norte de Minas (PANM): estudo da disponibilidade hídrica subterrânea do norte de Minas Gerais. Relatório de integração. 2019. Disponível em: https:// www.cprm.gov.br/publique/media/hidrologia/projetos/panm/relatorio_ integracao.pdf.

DIG - Distrito de Irrigação do Perímetro Gorutuba. Caracterização do Perímetro de Irrigação Gorutuba. 2013. Disponível em: http://www. dig.org.br/noticia/Caracterizacao-do-Perimetro-de-Irrigacao-Gorutubadistrito-do-gorutuba/31/. 
Utilização do algoritmo de árvore de decisão para mapeamento do uso e cobertura da terra da área de contribuição de uma barragem localizada na Bacia Hidrográfica do São Francisco

EMBRAPA - Empresa Brasileira de Pesquisa Agropecuária. Sistema brasileiro de classificação de solos. 2006. Disponível em: https://www. embrapa.br/solos/sibcs.

EMBRAPA - Empresa Brasileira de Pesquisa Agropecuária. Transferência de tecnologia florestal: cultivo de eucalipto em propriedades rurais: diversificação da produção e renda. 2014. Disponível em: https:// ainfo.cnptia.embrapa.br/digital/bitstream/item/121607/1/ApostilaSerie-TT-Eucalipto.pdf.

FEAM - Fundação Estadual do Meio Ambiente. Brasil, 2010. Disponível em: http://www.dps.ufv.br/?page_id=742.

Huete, A. R. 1988. A soil-adjusted vegetation index. Remote Sensing of Environment, 25, 295-309. Doi: https://doi.org/10.1016/00344257(88)90106-X.

INMET - Instituto Nacional de Meteorologia. Normais climatológicas do Brasil. Disponível em: https://portal.inmet.gov.br/servicos/normaisclimatol\%C3\%B3gicas.

Jensen, J. R. 2015. Introductory digital image processing: A remote sensing perspective. 4. ed. University of South Carolina, Pearson Education, USA.

Köppen, W. 1936. Das geographische System der Klimate. In: Handbuch der Klimatologie, KÖPPEN, W.; GEIGER, R. (Eds.), Gebruder Borntrager, Berlin, 1, 1-44, part C. Doi: http://koeppen-geiger.vu-wien.ac.at/pdf/ Koppen_1936.pdf.

Landis, J.; Koch, G. 1977. The measurement of observer agreement for categorical data, Washington, USA. Biometrics, 33:1, 159-174. Doi: https://doi.org/10.2307/2529310.

Leite, M. E.; Dias, L. S.; Rocha, A. M. 2015. Análise da ocupação no entorno da Barragem Bico da Pedra, no Município de Janaúba/MG. Caderno de Geografia, 25:44, 221-236. Doi: https://doi.org/10.5752/ P.2318-2962.2015v25n44p221.
Martins, E. M.; Fortes, J. D. N.; Ribeiro, G. P; Pereira, M. F. M. 2014. Utilização de sistema de informação geográfica como ferramenta para gestão do monitoramento da qualidade do ar na região metropolitana do Rio de Janeiro. Engenharia Agrícola e Ambiental, 19. Doi: https:// doi.org/10.1590/S1413-41522014019010001237.

Oliveira, W. F.; Sá, R. A.; Leite, M. E. 2016. Dinâmica do uso e ocupação do solo na área de drenagem do Reservatório de abastecimento do sistema Juramento/MG. Caminhos de Geografia, 17:57, 92-106. Doi: https://doi.org/10.14393/RCG175706.

Rosa, R. 2005. Geotecnologias na Geografia aplicada. Revista do Departamento de Geografia, 16, 81-90. Doi: https://doi.org/10.7154/ RDG.2005.0016.0009.

Rouse, J. W.; Haas Junior, R. H.; Sheel, J. A.; Deering, D. W. 1973. Monitoring vegetation systems in the great plains with ERTS. In: Earth resources technology satellite-1 symposium, 3., Washington. Anais... Washington: NASA, 1, 309-317. Disponível em: https://ntrs.nasa.gov/ citations/19740022614.

Santos, A. B. Petronzio, J. A. C. Mapeamento de uso e ocupação do solo do município de Uberlândia-MG utilizando técnicas de Geoprocessamento. In: XV Simpósio Brasileiro de Sensoriamento Remoto, 15., Curitiba. Anais... Curitiba: INPE. 1, 6185-6192. Disponível em: http://marte. sid.inpe.br/col/dpi.inpe.br/marte/2011/07.28.18.42/doc/p0210.pdf.

Sousa Junior, J. G. A.; Demattê, J. A. M.; Genú, A. M. 2008. Comportamento espectral dos solos na paisagem a partir de dados coletados por sensores terrestre e orbital. Revista Brasileira de Ciência do Solo, 32:2, 727-738. Doi: http://dx.doi.org/10.1590/S010006832008000200027.

Yang, C.; Wu, G.; Ding, K.; Shi, T.; Li, Q.; Wang, J. 2017. Improving land use/land cover classification by integrating pixel unmixing and decision tree methods. Remote Sensing, 9:12. Doi: https://doi.org/10.3390/ rs9121222.

ZEE - Zoneamento Ecológico Econômico. Mapeamento da Cobertura Vegetal, 2009. Disponível em: http://idesisema.meioambiente.mg.gov. br/. 